\title{
Glucuronidation as a metabolic barrier against zearalenone in rat everted intestine
}

\author{
Takahiro IEKO ${ }^{1)}$, Sumire INOUE ${ }^{1)}$, Yume INOMATA ${ }^{1)}$, Hiroki INOUE ${ }^{1,2)}$, \\ Jumpei $\mathrm{FUJIKI}^{1)}$ and Hidetomo IWANO ${ }^{1) *}$
1)Laboratory of Veterinary Biochemistry, Department of Veterinary Medicine, Rakuno Gakuen University, Ebetsu, Hokkaido 069-8501, Japan \\ ${ }^{2)}$ Nihon Doubutsu Tokushu Shindan Co., Ltd., Eniwa, Hokkaido 061-1374, Japan
}

J. Vet. Med. Sci.

82(2): 153-161, 2020

doi: 10.1292/jvms.19-0570

Received: 17 October 2019

Accepted: 28 November 2019

Advanced Epub:

16 December 2019
ABSTRACT. Zearalenone (ZON), produced by Fusarium fungi, exhibits estrogenic activity. Livestock can be exposed to ZON orally through contaminating feeds such as cereals, leading to reproductive disorders such as infertility and miscarriage via endocrine system disruption. However, the details of ZON metabolism remain unclear, and the mechanism of its toxicity has not been fully elucidated. In this study, we investigated the kinetics of ZON absorption and metabolism in rat segmented everted intestines. ZON absorption was confirmed in each intestine segment $60 \mathrm{~min}$ after application to the mucosal buffer at $10 \mu \mathrm{M}$. Approximately half of the absorbed ZON was metabolized to a-zearalenol, which tended to be mainly glucuronidated in intestinal cells. In the proximal intestine, most of the glucuronide metabolized by intestinal cells was excreted to the mucosal side, suggesting that the intestine plays an important role as a first drug metabolism barrier for ZON. However, in the distal intestine, ZON metabolites tended to be transported to the serosal side. Glucuronide transported to the serosal side could be carried via the systemic circulation to the local tissues, where it could be reactivated by deconjugation. These results are important with regard to the mechanism of endocrine disruption caused by ZON.

KEY WORDS: absorption, everted intestine, glucuronidation, metabolism, zearalenone

Zearalenone (ZON) is a nonsteroidal estrogen-like mycotoxin produced by Fusarium species [7]. ZON has been shown to disrupt reproductive processes by mimicking the action of 17ß-estradiol [42]. ZON is a common contaminant of grains such as corn and wheat worldwide $[1,26]$. Due to its high thermal stability, ZON is not degraded by heat treatment during processing, resulting in reports of ZON contamination of foods produced from grains [51]. ZON contamination of livestock feeds is also a problem, particularly feeds for pigs.

ZON primarily affects the reproductive organs in mammals, resulting in reproductive disorders such as uterine hypertrophy, vulva vaginitis, infertility, and miscarriage [56]. Delay in reaching sexual maturity and disruption of implantation have been demonstrated experimentally in rodents treated with ZON [24, 54]. After oral exposure to ZON, the mycotoxin is rapidly absorbed in the gastrointestinal tract. ZON uptake is estimated to be $80-85 \%$, and the mycotoxin and its modified forms can be detected in blood after administration [35]. ZON and its major derivatives are shown in Fig. 1 [33].

$\mathrm{ZON}$ is metabolized to $\alpha$ - and $\beta$-zearalenol (ZOL) via enzymatic reactions mediated by $3 \alpha$ - and $3 \beta$-hydroxy-5-steroid dehydrogenases (HSDs). The resultant $\alpha$ - and $\beta$-ZOL are metabolized via double bond reduction to $\alpha$ - and $\beta$-zearalanol (ZAL), respectively. In an alternative pathway, $Z O N$ is reversibly reduced to zearalanone (ZAN) $[33,36]$. These metabolites are reactive and exhibit more-potent estrogenic activity than ZON (relative estrogen potency: $\alpha$-ZAL $>\alpha-Z O L>\beta-Z A L>Z O N>\beta-Z O L$ ) $[17,33]$. Activated ZON derivatives undergo conjugation reactions catalyzed by UDP-glucuronosyltransferase (UGT) 1 A1 and UGT1A8 [40]. The resulting glucuronides have low estrogenic activity and are eliminated from the body via the urine or feces $[10,28,33]$. The alternative metabolism of ZON (i.e., metabolic activation and conjugation) is associated with various potential adverse reproductive effects. To elucidate the mechanism of ZON-induced adverse effects on target organs, therefore, it is essential to clarify the metabolism and disposition of ZON during passage through the gastrointestinal tract.

Binder et al. suggested that biotransformation of ZON occurs in the intestinal wall during absorption [2]. However, details regarding the actual and dynamic behavior of the compound during absorption within the intestinal wall remain unclear. Because there are gender differences in the effects of ZON, especially reproductive disorders during pregnancy [24], we used a rat evertedintestine model to examine the metabolism and disposition of ZON, focused on gender and pregnancy differences, in the intestine,

*Correspondence to: Iwano, H.: h-iwano@rakuno.ac.jp

(Supplementary material: refer to PMC https://www.ncbi.nlm.nih.gov/pmc/journals/2350/)

(O2020 The Japanese Society of Veterinary Science

This is an open-access article distributed under the terms of the Creative Commons Attribution Non-Commercial No Derivatives (by-nc-nd) License. (CC-BY-NC-ND 4.0: https://creativecommons.org/licenses/by-nc-nd/4.0/) 


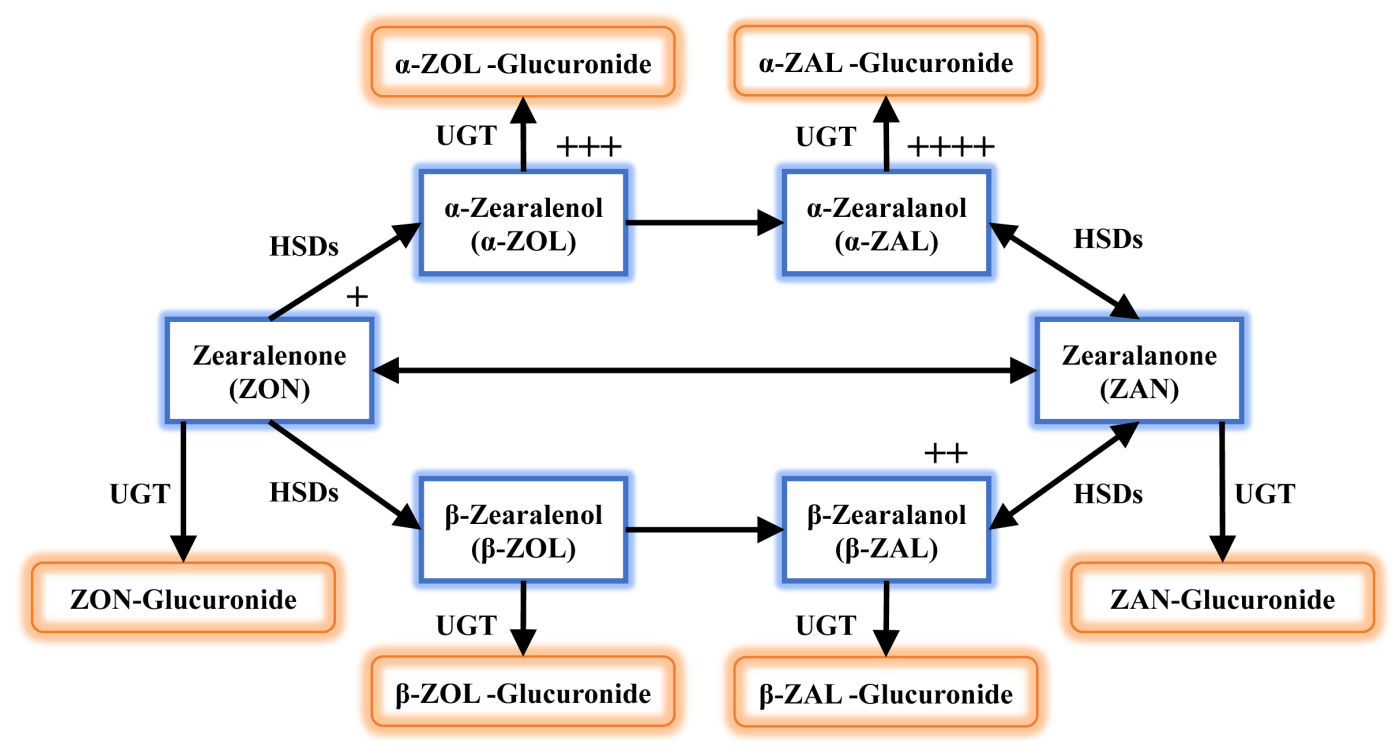

Fig. 1. Schematic representation of the zearalenone metabolic pathway. Zearalenone (ZON) metabolism in mammals involves reduction catalyzed by hydroxysteroid dehydrogenase (HSDs), and conjugation catalyzed by UDP-glucuronosyltransferase (UGT). The plus sign indicates the relative estrogenic potency of the compound. The order is $\alpha$-zearalanol $(\alpha-Z A L)>\alpha$-zearalenol $(\alpha$-ZOL $)>\beta$-zearalanol $(\beta-\mathrm{ZAL})>\mathrm{ZON}>\beta$-zearalenol $(\beta-\mathrm{ZOL})$.

which functions as the first barrier through xenobiotic metabolism. In this rat everted-intestinal model, it is possible to track the mucosal excretion and serosal transport of compounds over time by site of the intestine [13].

\section{MATERIALS AND METHODS}

\section{Chemicals}

ZON was purchased from Funakoshi Co. (Tokyo, Japan); high-performance liquid chromatography (HPLC)-grade methanol was purchased from Wako Pure Chemical Industries (Osaka, Japan); and $\beta$-glucuronidase was purchased from Sigma-Aldrich (St. Louis, MO, USA). Zearalenone glucuronide (ZON-GA) and zearalenol glucuronide (ZOL-GA), purified from rat bile after perfusion of the liver with ZON, were quantified by HPLC using the difference between $\beta$-glucuronidase-treated and untreated samples and used as standards [14].

\section{Ethics statement}

This study was carried out in strict accordance with the recommendations in the Guide for the Care and Use of Laboratory Animals of the U.S. National Institutes of Health. The protocol was approved by the Committee on the Ethics of Animal Experiments of the Rakuno Gakuen University (permit number: VH18A15). All surgeries were performed under isoflurane anesthesia, and every effort was made to minimize animal suffering.

\section{Animals}

Male (330-400 g), nonpregnant female (240-280 g), or pregnant female (270-340 g at gestation day 18-19) Sprague-Dawley rats (8 to 10 weeks old) were purchased from Sankyo Lab Co. (Tokyo, Japan) and used in all experiments. The rats were fed, housed, and allowed to adapt to their environment for 1 week before they were used in experiments. A total of 18 rats were used (6 males, 6 nonpregnant females, and 6 pregnant females).

\section{Preparation of everted intestine}

Krebs Ringer's bicarbonate buffer $\left(\mathrm{NaCl}, 110 \mathrm{mM} ; \mathrm{KCl}, 5 \mathrm{mM} ; \mathrm{MgCl}_{2}, 1.2 \mathrm{mM} ; \mathrm{CaCl}_{2}, 2.5 \mathrm{mM} ; \mathrm{NaHCO}_{3}, 25 \mathrm{mM}\right.$ and glucose, $10 \mathrm{mM}$ ) was used in all experiments. The solution was aerated with $95 \% \mathrm{O}_{2} / 5 \% \mathrm{CO}_{2}$, and the pH was adjusted to 7.4. After euthanasia by exsanguination under anesthesia, the jejunum, ileum, and colon were collected from each animal. The bowels were excised and prepared according to a modification of a previously described segmentation and eversion method [15]. Briefly, with the exception of the duodenum, the excised small intestine was lavaged and divided into three sections of equal length. The distal portion of each section was excised and trimmed to $10 \mathrm{~cm}$ and designated as segments I, II, and III in distal order, with segment I from the jejunum and segment III from the distal ileum (Fig. 2A). In the same manner, the colon (segment IV) was excised, washed, and trimmed to a final segment length of $10 \mathrm{~cm}$ taken from the distal end.

The four trimmed segments were turned inside out and affixed to a polyethylene tube containing mucosal buffer solution 
A

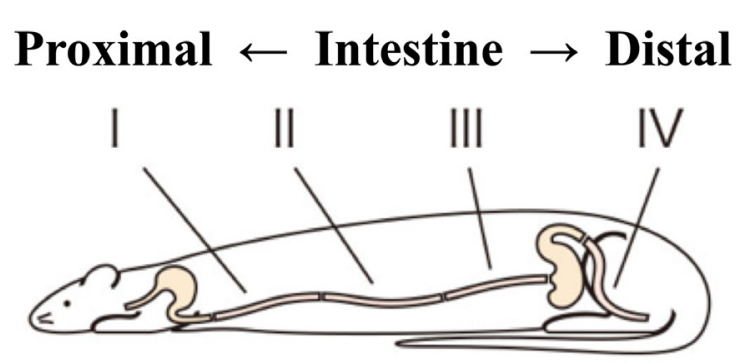

B

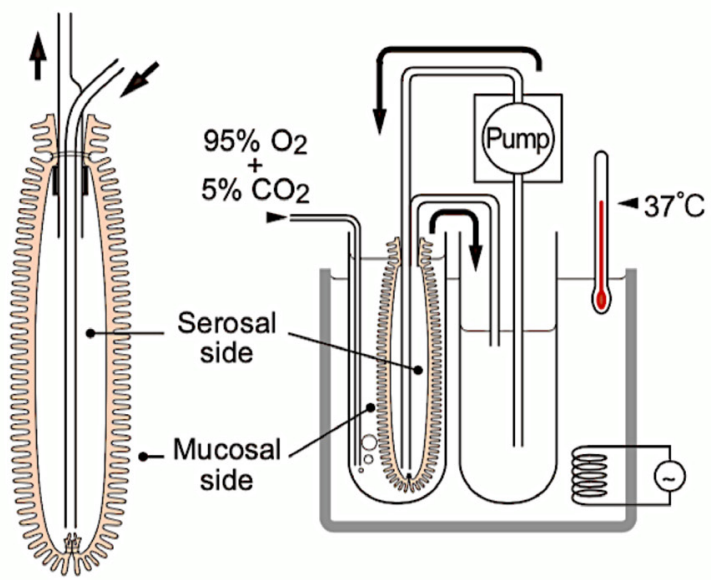

Fig. 2. Schematic illustration of the everted intestine model. (A) The small intestine, excluding the duodenum, was divided into three equal parts, and the distal part was excised and the length adjusted to $10 \mathrm{~cm}$. The parts were then designated I, II, and III in distal order. In the same manner, the colon was excised and adjusted to $10 \mathrm{~cm}$, taken from the distal end. (B) The everted intestine was affixed to a polyethylene tube. Serosal buffer was circulated at $5 \mathrm{ml} / \mathrm{min}$ using a pump. Zearalenone $(10 \mu \mathrm{M})$ was added to the mucosal buffer. The water temperature was maintained at $37^{\circ} \mathrm{C}$, and the tissue was aerated with $95 \% \mathrm{O}_{2} / 5 \% \mathrm{CO}_{2}$.

Table 1. Optimized multiple reaction monitoring parameters

\begin{tabular}{llccc}
\hline \multicolumn{1}{c}{ Analyte } & & $m / z$ & $\mathrm{RT}^{\text {a) }}(\mathrm{min})$ & $\mathrm{CE}^{\text {b) }}(\mathrm{V})$ \\
\hline Zearalenone & ZON & $317>273$ & 7.58 & 21 \\
Zearalanone & ZAN & $319>205$ & 7.52 & 23 \\
$\alpha$-Zearalenol & $\alpha$-ZOL & $319>160$ & 7.50 & 32 \\
$\beta$-Zearalenol & $\beta-Z O L$ & $319>160$ & 7.30 & 32 \\
$\alpha$-Zearalanol & $\alpha-Z A L$ & $321>277$ & 7.41 & 24 \\
$\beta$-Zearalanol & $\beta-Z A L$ & $321>277$ & 7.24 & 24 \\
Zearalenone glucuronide & ZON-GA & $493>175$ & 6.57 & 19 \\
$\alpha$-Zearalenol glucuronide & $\alpha-Z O L-G A$ & $495>319$ & 6.62 & 29 \\
$\beta$-Zearalenol glucuronide & $\beta-Z O L-G A$ & $495>319$ & 6.32 & 29 \\
Zearalenone diglucuronide & ZON-GA/GA & $669>493$ & 5.75 & 23 \\
\hline
\end{tabular}

a) RT, retention time; b) CE, collision energy.

$(25 \mathrm{~m} l)$. Serosal buffer solution $(25 \mathrm{~m} l)$ was pumped through the everted bowels using a tube pump MP-32N (EYELA, Tokyo, Japan) at $5 \mathrm{ml} / \mathrm{min}$ via polyethylene tubes (Fig. 2B). ZON was added to the mucosal buffer solution at a concentration of $10 \mu \mathrm{M}$, and reaction products were collected independently from the serosal and mucosal sides at $0,20,40$, and 60 min after the addition of each compound. In this rat everted-intestinal model as described, sufficient metabolic kinetics could not be analyzed when the substrate concentration was below this level [13]. Moreover, this level of ZON is known not to affect cell viability in experiments in vitro experiment [42].

\section{Liquid chromatography-tandem mass spectrometry (LC-MS/MS) analysis of reaction products}

Mucosal and serosal samples were filtered using a disposable disk filter (HLC-DISK3; Kanto Chemical Co.) and stored at $-80^{\circ} \mathrm{C}$ until analysis. The samples were then analyzed using an LC-MS/MS system (Shimadzu, Ktoto, Japan) equipped with an electrospray ionization source and operated in negative mode. Mobile phases A and B consisted of methanol/water/acetic acid (5:95:0.05) with $10 \mathrm{mM}$ ammonium acetate and 100\% methanol, respectively. Gradient elution was performed as follows: 0-8 $\min (0-100 \%$ B), 8-10 min $(100 \%$ B). Samples were resolved on a Triart C18 reversed-phase column $(2.1 \times 150 \mathrm{~mm}$; YMC Co., Tokyo, Japan) and detected using multiple reaction monitoring mode. The precursor and product ions are shown in Table 1.

${ }^{13} \mathrm{C}-\mathrm{ZON}$ was used as an internal standard to quantify $\mathrm{ZON}$ and its derivatives.

\section{Statistical analysis}

All data are expressed as the mean \pm S.D. of six independent experiments. Mann-Whitney $U$ test was applied to compare the quantitative variables by JMP 14.2 (SAS institute, Japan). In all the statistical tests, differences were considered significant if the $P$ value was equal to or less than 0.05 . 


\section{RESULTS}

\section{Zearalenone absorption and transport}

Upon application of ZON $(10 \mu \mathrm{M})$ to the mucosal side of the everted intestine, the fluid concentration of ZON decreased over the incubation period. The rate of disappearance of $\mathrm{ZON}$ from the mucosal compartment was estimated at approximately 100-200 nmol/hr in all segments (Fig. 3). In male and pregnant female rats, the disappearance tended to be diminished in the distal segment of the small intestine (segment III), however the disappearance of ZON was almost the same at any parts of the female rat intestinal segments.

On the serosal side of the everted intestine, a small amount of ZON was detected. The amount of ZON transported from the mucosal side to the serosal side was extremely low (approximately $2 \mathrm{nmol}$ over $60 \mathrm{~min}$ of incubation) even in the distal colon, where the highest absorption of ZON was observed (Fig. 3 and Supplementary Fig. 1A).

\section{Absorption and excretion of ZON metabolites}

The present data demonstrate the transport of a small amount of ZON from the mucosal side to the serosal side of the everted intestine, despite the considerable amount of ZON absorbed from the mucosal fluid (Fig. 3). In a recent study, ZON was shown to be metabolized to ZON-GA, $\alpha-Z O L, \beta-Z O L, \alpha-Z O L-G A, \beta-Z O L-G A, Z O N-G A$, and ZAN [33]. Therefore, the excretion of ZON metabolites from the intestinal segments was examined.

After 60 min of incubation, $\alpha$-ZOL, ZON-GA, and $\alpha$-ZOL-GA were detected in the mucosal and serosal buffers and quantified. ZAN, $\beta$-ZOL, $\beta$-ZOL-GA, and zearalenone diglucuronide were also detected, but the amounts were too low to quantify.

Excretion of $\alpha-Z O L$ (which is reduced at the $7 \alpha$ position of ZON by HSDs) to the mucosal side was observed in each segment. $\alpha$-ZOL mucosal excretion tended to be higher in the male proximal intestine (segment I) and in male and pregnant female distal intestine (segments III and IV), but the amount was very small, at most approximately 0.3 nmol (Fig. 4 and Supplementary Fig. 1B).

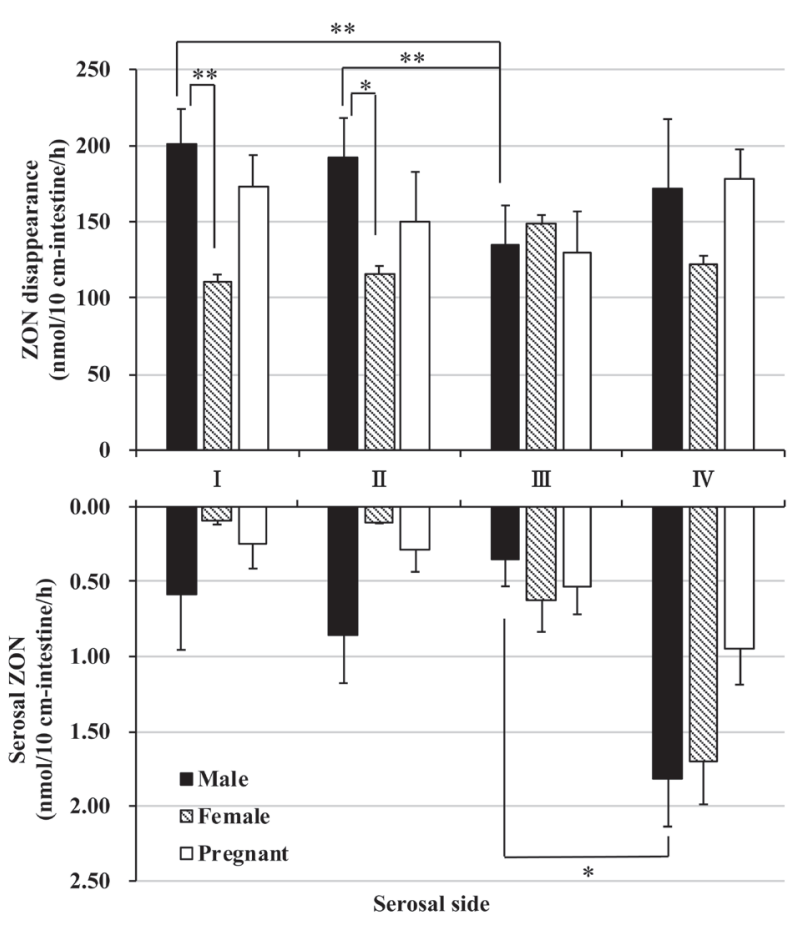

Fig. 3. Absorption and disposition of zearalenone in rat everted intestine. Upper graph shows the amount of zearalenone (ZON) that disappeared from the mucosal side within $60 \mathrm{~min}$ of incubation. ZON $(10 \mu \mathrm{M})$ was added to the mucosal buffer solution of each segment. The amount of $\mathrm{ZON}$ was determined by subtracting the final amount of ZON in the mucosal buffer solution after a 60-min incubation. I, II, and III indicate the intestinal sites in distal order from the ligament of Trietz, and IV indicates the colon. Bottom graph shows the amount of unconjugated ZON transported to the serosal side within $60 \mathrm{~min}$ of incubation. ZON was added to the mucosal buffer solution of each segment at a concentration of $10 \mu \mathrm{M}$. Segments I, II, and III are distal from the jejunum and ileum, and IV is the colon. $* P<0.05$, ** $P<0.01$.

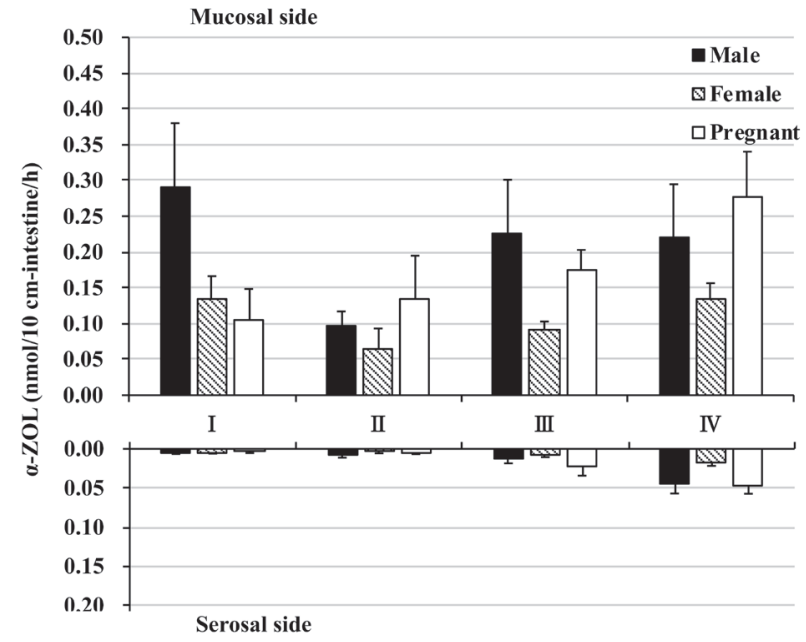

Fig. 4. $\alpha$-Zearalenol in rat everted intestine. The amount of $\alpha$-zearalenol ( $\alpha$-ZOL) excretion to the mucosal side (top graph) and transported to the serosal side (bottom graph) within 60 min of incubation. Zearalenone was added to the mucosal buffer solution of each segment at a concentration of $10 \mu \mathrm{M}$. Segments I, II, and III are from the jejunum and ileum in distal order, and IV is the colon. 


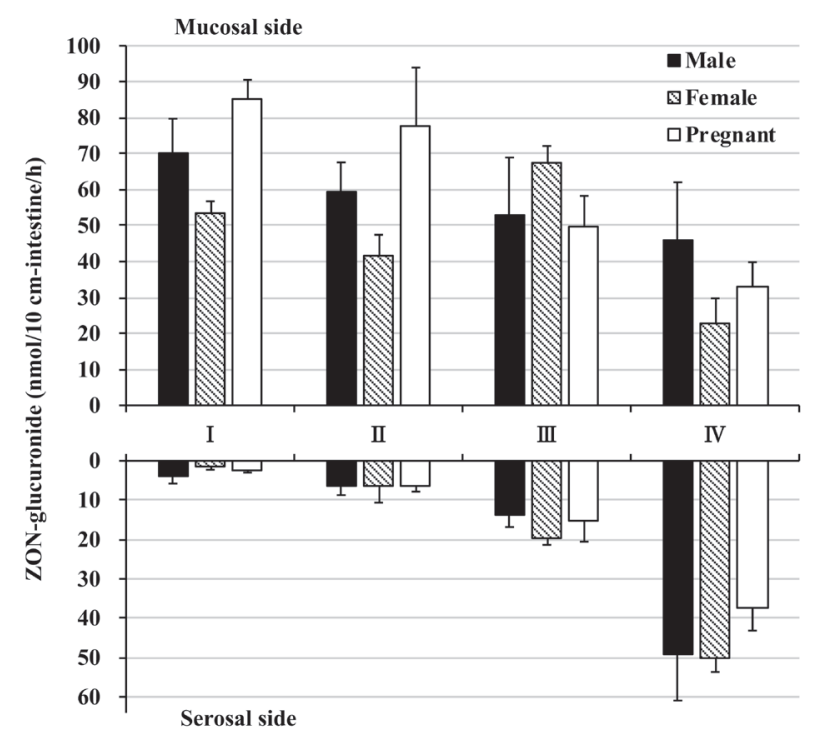

Fig. 5. Glucuronidation of zearalenone in rat everted intestine. The amount of zearalenone (ZON) glucuronidated and excreted to the mucosal side (top graph) and transported to the serosal side (bottom graph) within 60 min of incubation. ZON was added to the mucosal buffer solution of each segment at a concentration of $10 \mu \mathrm{M}$. Segments I, II, and III are from the jejunum and ileum in distal order, and IV is the colon.

In contrast, $\alpha-Z O L$ transport to the serosal side reached a maximum of approximately $0.05 \mathrm{nmol}$ in the colon, an amount that was even less than observed with mucosal excretion; $\alpha$-ZOL transport to the mucosal side was approximately 6 times greater than transport to the serosal side (Fig. 4 and Supplementary Fig. 1C).

Most of the absorbed ZON was not transported to the serosal side in its unmodified form nor excreted or transported as reduced $\alpha-Z O L$. Therefore, we investigated the possibility that $\mathrm{ZON}$ was conjugated in the intestine. In all intestinal segments, ZON-GA and $\alpha$-ZOL-GA, the glucuronidated forms of $Z O N$ and $\alpha-Z O L$, were detected in large amounts. ZON-GA mucosal excretion reached a maximum of 85.39 $\mathrm{nmol}$ in the pregnant female proximal intestine and a minimum of $22.55 \mathrm{nmol}$ in the female colon (Fig. 5 and Supplementary Fig. 1D). In contrast, ZON-GA serosal transport reached a maximum of 49.94 $\mathrm{nmol}$ in the female colon and a minimum of $1.64 \mathrm{nmol}$ in the female proximal intestine (Fig. 5 and Supplementary Fig. 1E). The maximum $\alpha$-ZOL-GA mucosal excretion was $60.02 \mathrm{nmol}$ in the pregnant female proximal intestine, and the minimum mucosal excretion was $20.09 \mathrm{nmol}$ in the female colon (Fig. 6 and Supplementary Fig. 1F). By comparison, the maximum $\alpha$-ZOL-GA serosal transport was $36.15 \mathrm{nmol}$ in the pregnant female colon, and the minimum serosal transport was $2.84 \mathrm{nmol}$ in the female proximal intestine (Fig. 6 and Supplementary Fig. 1G). Interestingly, the maximum amounts of ZON-GA and $\alpha$-ZOL-GA were secreted to the mucosal side of the intestine (segments I III), whereas in the colon, mucosal secretion of these glucuronides was reduced (Figs. 5 and 6). The lowest ZON-GA and $\alpha$-ZOL-GA secretion to the serosal side occurred in the proximal small intestine and increased with progression distally to the colon.

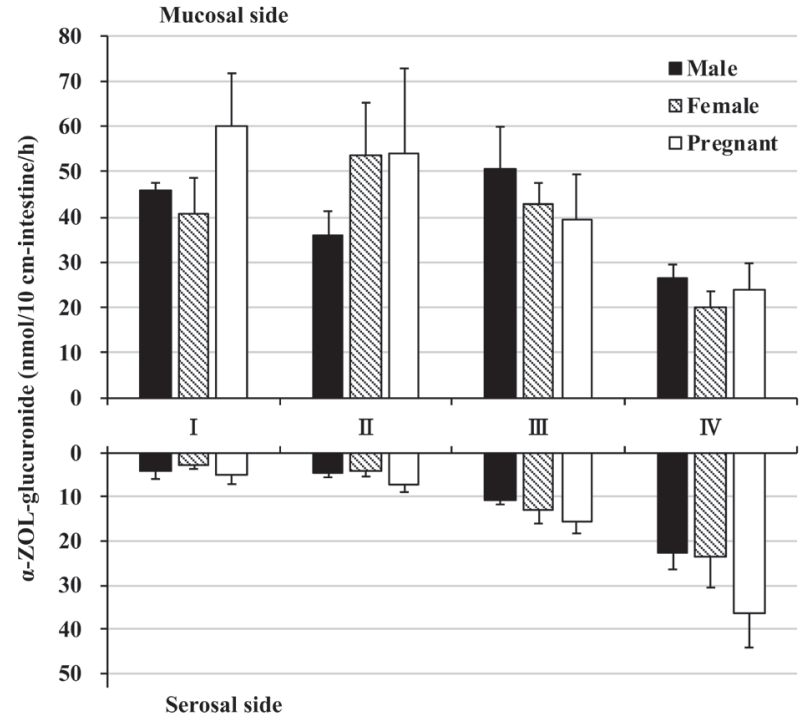

Fig. 6. Glucuronidation of $\alpha$-zearalenol in rat everted intestine. The amount of $\alpha$-zearalenol ( $\alpha$-ZOL) glucuronidated and excreted to the mucosal side (top graph) and transported to the serosal side (bottom graph) within $60 \mathrm{~min}$ of incubation. Zearalenone was added to the mucosal buffer solution of each segment at a concentration of $10 \mu \mathrm{M}$. Segments I, II, and III are from the jejunum and ileum in distal order, and IV is the colon.

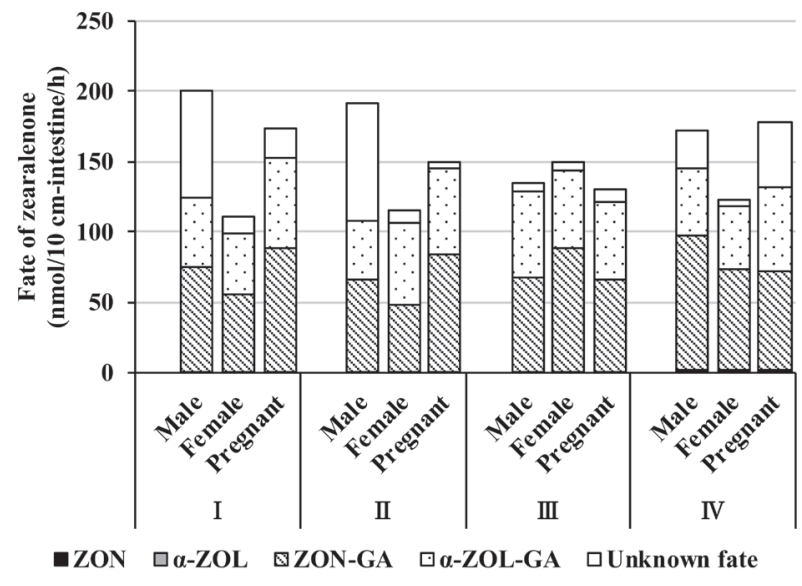

Fig. 7. Fate of zearalenone during a 60-min incubation in rat everted intestine. Total decrease in mucosal zearalenone (ZON) during a $60-\mathrm{min}$ incubation is depicted as the sum total of the fraction column in each intestinal segment. ZON, zearalenone transported to the serosal side; $\alpha$-ZOL, total $\alpha$-zearalenol transported to the serosal side and secreted to the mucosal side; ZON-GA, total zearalenone glucuronide transported to the serosal side and secreted to the mucosal side; $\alpha$-ZOL-GA, total $\alpha$-zearalenol glucuronide transported to the serosal side and secreted to the mucosal side; Unknown fate, zearalenone of unknown fate. Segments I, II, and III are distal from the jejunum and ileum, and IV is the colon.

\section{Fate of ZON at 60-min post-application}

The total balance of metabolism and transport after $60 \mathrm{~min}$ of incubation with ZON $(10 \mu \mathrm{M})$ was examined (Fig. 7). Most of the absorbed substrate was recovered $(>80 \%)$ in all segments in male, female, and pregnant females, with the exception of male small 
intestine segments I and II and pregnant female colon segment IV (Fig. 7). Absorbed ZON was rapidly metabolized to $\alpha$-ZOL, ZON-GA, and $\alpha$-ZOL-GA, based on the observation that absorbed ZON did not remain completely in its unmodified form in any of the intestine segments. Furthermore, each glucuronide was largely excreted to the mucosal side, but in the distal intestine, approximately half of each glucuronide was transported to the serosal side (Figs. 5 and 6).

\section{DISCUSSION}

ZON introduced orally must pass through the intestine before reaching the target organs, such as the reproductive system. To elucidate the mechanism responsible for the adverse effects of ZON, it is essential to clarify the fate of the compound in the intestine. However, details regarding the disposition of ZON during transport to the target organs remain unclear. In this study, we used a rat everted intestine model to elucidate the fate of $\mathrm{ZON}$ in the intestine, which due to metabolic activity functions as the first barrier to xenobiotics.

Our results indicate that most ZON absorbed from the mucosal side is reduced or glucuronidated in the intestinal cells. A small amount of unconjugated $\alpha-Z O L$ was detected, but ZAN and $\alpha-/ \beta-Z A L$ were not detected. It can be inferred that ZON is readily converted to ZOL in the rat intestine. ZON has also been reported to be hydroxylated primarily by CYP3A subfamily enzymes $[5,41]$. The CYP3A subfamily includes major drug-metabolizing enzymes in the intestine, and it was reported that CYP3A is also expressed in rat intestine $[11,23,31]$, but OH-ZON was not found in our results. ZON is also known to be converted to ZOL by HSDs [29]. Since high levels of $3 \alpha$-HSD mRNA were found in small intestine and colon [27], it seems that $3 \alpha-H S D$ is also involved in the ZON reduction reaction in the intestine. The examinations of the contribution rate of HSDs to ZOL metabolism represent interesting topics for future research. In our data, mucosal excretion of $\alpha-Z O L$ was low in each intestinal segment, but serosal transport was even lower. These data indicate that each intestine segment hardly passes $\alpha$-ZOL to the serosal side. Interestingly, the almost complete absence of detection of $\alpha$-ZOL suggests that $\alpha$-ZOL is immediately glucuronidated. In other words, by inactivating ZON via glucuronidation, the intestine functions as a barrier against absorption of foreign substances. Because the glucuronidation of ZON was particularly extensive in all intestinal segments (Fig. 7), UGT expression and activity are high in the intestine, and ZON and $\alpha$-ZOL likely undergo immediate glucuronidation. This suggests that the intestine functions as a barrier to ZON exposure. In general, enzymes of the UGT2B family glucuronidate steroid hormones [50]. UGT1A1, 1A7, $1 \mathrm{~A} 8$, and $2 \mathrm{~A} 3$ are highly expressed in the rodent intestine, especially the proximal intestine $[4,38]$. A study using human liver and intestinal microsomes reported that UGT1A1, 1A3, 1A8, and $2 \mathrm{~B} 7$ exhibited high activity against ZON [40]. Ugt2b mRNA accounts for approximately $80 \%$ of total Ugt mRNA in the rat liver, whereas Ugtla mRNA accounts for almost $90 \%$ of total Ugt mRNA in the rat small intestine [25]. UGT1A1 and 1A8 thus appear to be the most promising candidates responsible for ZON glucuronidation in the intestine.

More than half of the ZON absorbed in the small intestine in the present study was glucuronidated, and most of it was excreted to the mucosal side. This is consistent with the small intestinal defense mechanism against bisphenol A, an endocrine-disrupting chemical known to have estrogenic effects similar to ZON $[13,16]$. These data suggest that the proximal intestine plays a highly protective role, restricting the dissemination of xenobiotics and inactivating them via glucuronidation, thus restricting exposure to the active substances to the middle and distal parts of the intestines. ATP-dependent transporters, namely multidrug resistance associated proteins (MRPs), are known to be capable of mediating transmembrane excretion of a wide range of amphiphilic compounds, including estrogens and glucuronides [37, 52]. MRP2, which is localized in the apical domain of enterocytes, is distributed in the proximal intestine in rats, and MRP3, which is localized in the basal domain, is distributed in the colon [21, 32, 46]. Interestingly, in this study, the excretion of ZON-GA and $\alpha$-ZOL-GA in the apical direction and transport in the basal direction were consistent with the respective localization and distribution patterns of MRP2 and MRP3 in the rat intestine. From these data, we speculate that ZON-GA and $\alpha$-ZOL-GA are excreted and transported in the intestine by MRP2 and MRP3, respectively (Fig. 8).

It has been shown that the entire intestine functions as a barrier to xenobiotics, but there is also a risk of exposure. In this study, ZON and its metabolites were also transported to the serosal side. The level of ZON serosal transport observed in each intestinal segment was extremely low, and this seems not to be problematic because the experiment was performed using a large amount of ZON. However, compared with ZON, the amount of glucuronidated form transported to the serosal side was quite high, approximately 10 times greater in the small intestine and approximately 20 times greater in the colon. Some of the conjugates transported into the blood are known to be distributed to local organs without being excreted [16, 34]. Conjugates transported to local organs can reportedly cause adverse effects due to deconjugation and reactivation within the local organs. In our study, the serosal transport of ZON-GA and $\alpha$-ZOL-GA was particularly high in the colon. Anatomically, some of the compounds absorbed in the distal colon do not pass through the liver and circulate systemically without the first effect of the liver (Fig. 8). Therefore, it is possible that most of the ZON-GA and $\alpha$-ZOL-GA absorbed in the colon circulate through the body and are distributed in local organs, where they could be reactivated by deconjugation. In addition, the serosal transport of $\alpha$-ZOL-GA was comparable to that of ZON-GA. The estrogenic activity of $\alpha-Z O L$ is three times more potent than that of ZON [30]. This suggests that the blood absorption of $\alpha$-ZOL-GA in the colon plays an important role in the mechanism by which orally ingested ZON disrupts the endocrine system.

ZON-GA and $\alpha$-ZOL-GA excreted to the mucosal side in the proximal jejunum are thought to flow into the distal intestine with the digestive contents. ZON is also excreted in the bile after glucuronidation in the liver [53]. In general, glucuronides are likely to enter the enterohepatic circulation [39], suggesting that large amounts of ZON-GA and $\alpha-Z O L-G A$ flow into the distal intestine. In the colon, glucuronides can be deconjugated by enterobacterial $\beta$-glucuronidase [43]. Irinotecan, an antineoplastic drug, is 


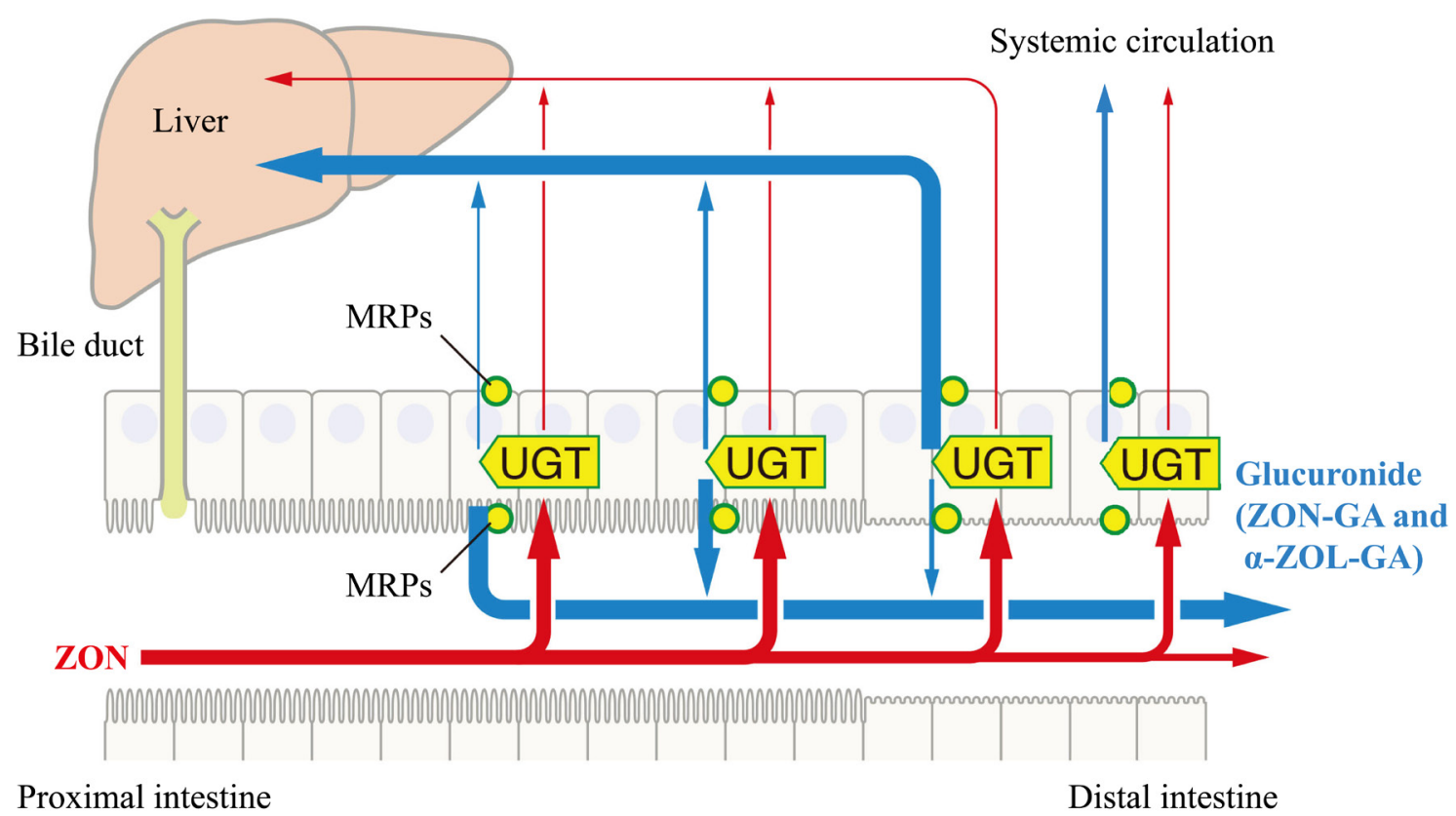

Fig. 8. Schematic figure of our hypothesis of intestine region-dependent metabolism and excretion of metabolites. Most of the zearalenone (ZON) absorbed in the intestine is glucuronidated to zearalenone glucuronide (ZON-GA) or $\alpha$-zearalenol glucuronide ( $\alpha$-ZOL-GA) by UDPglucuronosyltransferase (UGT). Then, in the proximal intestine, it is likely to be excreted mainly to the mucosal side via multidrug resistance associated proteins 2 (MRP2), while in the distal intestine, it is likely to be mainly transported to the serosal side via MRP3. Some slight ZON may be transported to the serosal side in its unmodified form. It is possible that some ZON and its glucuronide absorbed in the distal colon may circulate systemically without passing through the liver.

metabolized in the body by carboxylesterase to the active substance 7-ethyl-10-hydroxycamptothecin (SN-38). Thus, SN-38 exhibits antineoplastic activity [12]. SN-38 is glucuronidated by UGT in the liver and excreted in the bile as an inactive substance, SN-38 glucuronide (SN-38-GA) [45]. SN-38-GA excreted in bile is reportedly deconjugated by enterobacterial $\beta$-glucuronidase and reactivated [20]. Reactivated SN-38 can be resorbed in the distal intestine and damage mucosal tissues [49]. The behavior pattern of irinotecan in the body can be applied to ZON, with ZON-GA and $\alpha-Z O L-G A$ flowing to the distal intestine and being reactivated by deconjugation, with a risk of eventual reabsorption in the colon.

In this study, the rate of recovery of absorbed ZON in the proximal intestine was low. Ingestion of low doses of ZON in immature gilts reportedly leads to accumulation of ZON in the proximal intestine immediately after exposure [55]. As in pigs, it is possible that ingested ZON accumulates in the proximal jejunum in rats, but further investigation is necessary to confirm this possibility. Compared with the male distal intestine and the female proximal intestine, the male proximal intestine absorbed more ZON from the mucosal side, and there was a large amount of undetected material, indicating unknown fate. This suggests that ingested ZON follows different metabolic pathways in males and females, which could be associated with sex-specific differences in toxicity. In our results, although there were some slight gender differences in the absorption of ZON in the intestine, there was no significant difference in the kinetics of ZON-GA and $\alpha$-ZOL-GA, the main metabolite of ZON in the intestine, between male and female, and between pregnancy and non-pregnancy. It is known that the expression in the intestine of CYP3A, which is presumed to be involved in the reduction of ZON, is not different by gender [22]. In addition, transcriptional regulation of the UGT1A genes in jejunum and colon has been reported to be suppressed through estrogen receptor $\alpha(\mathrm{ER} \alpha)$ [18], but it is known that gender differences in UGT expression vary with species and strains [25]. It is also known that few Ugt genes are affected by pregnancy [48]. On the other hand, the expression in the liver of the enzymes involved in the metabolism of ZON and the transporters related to the transport of ZON metabolites has been reported to differ depending on the gender and presence or absence of pregnancy $[8,22,25,48]$. From these facts, the difference in ZON kinetics between male and female, and between pregnancy and non-pregnancy, is more likely to be affected by liver metabolism than intestine. Elucidation of the detailed metabolic kinetics of ZON in the liver, focusing on gender and pregnancy status, is interesting topic for future research.

The intestine is the first barrier to orally ingested xenobiotics. In investigating the effects of xenobiotics, it is important to track the fate of the target compound in the intestine before it enters the bloodstream. The present study revealed that $\mathrm{ZON}$ is metabolized to an inactive metabolite, ZON-GA or $\alpha-Z O L-G A$, in the proximal intestine and then excreted to the intestinal lumen (Fig. 8). This confirms that the intestine plays a significant defensive role against the dissemination of ZON in the body. However, the metabolites ZON-GA and $\alpha-Z O L-G A$ are absorbed into the blood primarily in the colon, suggesting that there is a risk of transport to local target organs. It would be interesting to investigate how ZON-GA and $\alpha$-ZOL-GA are subsequently metabolized and distributed after being absorbed into the blood in the intestine. Fully elucidating the mechanism of ZON toxicity is thus an 
important issue for future research. Additionally, ZON is known to be metabolized by plants and fungi, undergoing glycoside or sulfate conjugation $[19,44]$. These externally conjugated compounds are known as masked mycotoxins, and their presence as contaminants has been reported in food and feed products [3, 6, 47]. Although masked mycotoxins are harmless to mammals, considerable care is required to prevent their conversion to the parental mycotoxins after ingestion and further biotransformation into unidentified metabolites [9]. Future research should also investigate the behavior of these compounds.

\section{REFERENCES}

1. Balendres, M. A. O., Karlovsky, P. and Cumagun, C. J. R. 2019. Mycotoxigenic fungi and mycotoxins in agricultural crop commodities in the philippines: A review. Foods 8: 8. [Medline] [CrossRef]

2. Binder, S. B., Schwartz-Zimmermann, H. E., Varga, E., Bichl, G., Michlmayr, H., Adam, G. and Berthiller, F. 2017. Metabolism of zearalenone and its major modified forms in pigs. Toxins (Basel) 9: 9. [Medline] [CrossRef]

3. Boevre, M. D., Mavungu, J. D. D., Landschoot, S., Audenaert, K., Eeckhout, M., Maene, P., Haesaert, G. and Saeger, S. D. 2012. Natural occurrence of mycotoxins and their masked forms in food and feed products. World Mycotoxin J. 5: 207-219. [CrossRef]

4. Bolling, B. W., Court, M. H., Blumberg, J. B. and Chen, C. Y. 2011. Microsomal quercetin glucuronidation in rat small intestine depends on age and segment. Drug Metab. Dispos. 39: 1406-1414. [Medline] [CrossRef]

5. Bravin, F., Duca, R. C., Balaguer, P. and Delaforge, M. 2009. In vitro cytochrome p450 formation of a mono-hydroxylated metabolite of zearalenone exhibiting estrogenic activities: possible occurrence of this metabolite in vivo. Int. J. Mol. Sci. 10: 1824-1837. [Medline] [CrossRef]

6. Broekaert, N., Devreese, M., De Baere, S., De Backer, P. and Croubels, S. 2015. Modified Fusarium mycotoxins unmasked: From occurrence in cereals to animal and human excretion. Food Chem. Toxicol. 80: 17-31. [Medline] [CrossRef]

7. Caldwell, R. W., Tuite, J., Stob, M. and Baldwin, R. 1970. Zearalenone production by Fusarium species. Appl. Microbiol. 20: 31-34. [Medline]

8. Cao, J., Stieger, B., Meier, P. J. and Vore, M. 2002. Expression of rat hepatic multidrug resistance-associated proteins and organic anion transporters in pregnancy. Am. J. Physiol. Gastrointest. Liver Physiol. 283: G757-G766. [Medline] [CrossRef]

9. Freire, L. and Sant'Ana, A. S. 2018. Modified mycotoxins: An updated review on their formation, detection, occurrence, and toxic effects. Food Chem. Toxicol. 111: 189-205. [Medline] [CrossRef]

10. Frizzell, C., Uhlig, S., Miles, C. O., Verhaegen, S., Elliott, C. T., Eriksen, G. S., Sørlie, M., Ropstad, E. and Connolly, L. 2015. Biotransformation of zearalenone and zearalenols to their major glucuronide metabolites reduces estrogenic activity. Toxicol. In Vitro 29: 575-581. [Medline] [CrossRef]

11. Guengerich, F. P. 1999. Cytochrome P-450 3A4: regulation and role in drug metabolism. Annu. Rev. Pharmacol. Toxicol. 39: 1-17. [Medline] [CrossRef]

12. Humerickhouse, R., Lohrbach, K., Li, L., Bosron, W. F. and Dolan, M. E. 2000. Characterization of CPT-11 hydrolysis by human liver carboxylesterase isoforms hCE-1 and hCE-2. Cancer Res. 60: 1189-1192. [Medline]

13. Inoue, H., Yuki, G., Yokota, H. and Kato, S. 2003. Bisphenol A glucuronidation and absorption in rat intestine. Drug Metab. Dispos. 31: 140-144. [Medline] [CrossRef]

14. Inoue, H., Yokota, H., Makino, T., Yuasa, A. and Kato, S. 2001. Bisphenol a glucuronide, a major metabolite in rat bile after liver perfusion. Drug Metab. Dispos. 29: 1084-1087. [Medline]

15. Inoue, H., Yokota, H., Taniyama, H., Kuwahara, A., Ogawa, H., Kato, S. and Yuasa, A. 1999. 1-Naphthol beta-D-glucuronide formed intraluminally in rat small intestine mucosa and absorbed into the colon. Life Sci. 65: 1579-1588. [Medline] [CrossRef]

16. Iwano, H., Inoue, H., Nishikawa, M., Fujiki, J. and Yokota, H. 2018. Biotransformation of bisphenol a and its adverse effects on the next generation. Endocr. Disruptors (Austin): 63. [CrossRef]

17. Jefferson, W. N., Padilla-Banks, E., Clark, G. and Newbold, R. R. 2002. Assessing estrogenic activity of phytochemicals using transcriptional activation and immature mouse uterotrophic responses. J. Chromatogr. B Analyt. Technol. Biomed. Life Sci. 777: 179-189. [Medline] [CrossRef]

18. Kalthoff, S., Winkler, A., Freiberg, N., Manns, M. P. and Strassburg, C. P. 2013. Gender matters: estrogen receptor alpha (ER $\alpha)$ and histone deacetylase (HDAC) 1 and 2 control the gender-specific transcriptional regulation of human uridine diphosphate glucuronosyltransferases genes (UGT1A). J. Hepatol. 59: 797-804. [Medline] [CrossRef]

19. Kamimura, H. 1986. Conversion of zearalenone to zearalenone glycoside by Rhizopus sp. Appl. Environ. Microbiol. 52: 515-519. [Medline]

20. Kaneda, N. and Yokokura, T. 1990. Nonlinear pharmacokinetics of CPT-11 in rats. Cancer Res. 50: 1721-1725. [Medline]

21. Klaassen, C. D. and Aleksunes, L. M. 2010. Xenobiotic, bile acid, and cholesterol transporters: function and regulation. Pharmacol. Rev. 62: 1-96. [Medline] [CrossRef]

22. Kobayashi, K., Abe, C., Endo, M., Kazuki, Y., Oshimura, M. and Chiba, K. 2017. Gender difference of hepatic and intestinal cyp3a4 in cyp3ahumanized mice generated by a human chromosome-engineering technique. Drug Metab. Lett. 11: 60-67. [Medline] [CrossRef]

23. Komura, H. and Iwaki, M. 2011. In vitro and in vivo small intestinal metabolism of CYP3A and UGT substrates in preclinical animals species and humans: species differences. Drug Metab. Rev. 43: 476-498. [Medline] [CrossRef]

24. Kunishige, K., Kawate, N., Inaba, T. and Tamada, H. 2017. Exposure to zearalenone during early pregnancy causes estrogenic multitoxic effects in mice. Reprod. Sci. 24: 421-427. [Medline] [CrossRef]

25. Kutsukake, T., Furukawa, Y., Ondo, K., Gotoh, S., Fukami, T. and Nakajima, M. 2019. Quantitative analysis of udp-glucuronosyltransferase ugtla and ugt2b mrna expression in the rat liver and small intestine: Sex and strain differences. Drug Metab. Dispos. 47: 38-44. [Medline] [CrossRef]

26. Li, M., Yang, C., Mao, Y., Hong, X. and Du, D. 2019. Zearalenone contamination in corn, corn products, and swine feed in china in $2016-2018$ as assessed by magnetic bead immunoassay. Toxins (Basel) 11: 11. [Medline] [CrossRef]

27. Lin, H. K., Jez, J. M., Schlegel, B. P., Peehl, D. M., Pachter, J. A. and Penning, T. M. 1997. Expression and characterization of recombinant type 23 alpha-hydroxysteroid dehydrogenase (HSD) from human prostate: demonstration of bifunctional 3 alpha/17 beta-HSD activity and cellular distribution. Mol. Endocrinol. 11: 1971-1984. [Medline]

28. Malekinejad, H., Maas-Bakker, R. F. and Fink-Gremmels, J. 2005. Bioactivation of zearalenone by porcine hepatic biotransformation. Vet. Res. 36 799-810. [Medline] [CrossRef]

29. Malekinejad, H., Van Tol, H. T., Colenbrander, B. and Fink-Gremmels, J. 2006. Expression of 3alpha- and 3beta-hydroxy steroid dehydrogenase mRNA in COCs and granulosa cells determines Zearalenone biotransformation. Toxicol. In Vitro 20: 458-463. [Medline] [CrossRef]

30. Mirocha, C. J., Pathre, S. V. and Robison, T. S. 1981. Comparative metabolism of zearalenone and transmission into bovine milk. Food Cosmet. Toxicol. 19: 25-30. [Medline] [CrossRef] 
31. Mitschke, D., Reichel, A., Fricker, G. and Moenning, U. 2008. Characterization of cytochrome P450 protein expression along the entire length of the intestine of male and female rats. Drug Metab. Dispos. 36: 1039-1045. [Medline] [CrossRef]

32. Mottino, A. D., Hoffman, T., Jennes, L., Cao, J. and Vore, M. 2001. Expression of multidrug resistance-associated protein 2 in small intestine from pregnant and postpartum rats. Am. J. Physiol. Gastrointest. Liver Physiol. 280: G1261-G1273. [Medline] [CrossRef]

33. Mukherjee, D., Royce, S. G., Alexander, J. A., Buckley, B., Isukapalli, S. S., Bandera, E. V., Zarbl, H. and Georgopoulos, P. G. 2014. Physiologically-based toxicokinetic modeling of zearalenone and its metabolites: application to the Jersey girl study. PLoS One 9: e113632. [Medline] [CrossRef]

34. Nishikawa, M., Iwano, H., Yanagisawa, R., Koike, N., Inoue, H. and Yokota, H. 2010. Placental transfer of conjugated bisphenol A and subsequent reactivation in the rat fetus. Environ. Health Perspect. 118: 1196-1203. [Medline] [CrossRef]

35. Olsen, M. 1989. Fusarium: Mycotoxins, Taxonomy and Pathogenicity. pp. 167-177. Elsevier Amsterdam, Amsterdam.

36. Olsen, M., Pettersson, H. and Kiessling, K. H. 1981. Reduction of zearalenone to zearalenol in female rat liver by 3 alpha-hydroxysteroid dehydrogenase. Acta Pharmacol. Toxicol. (Copenh.) 48: 157-161. [Medline] [CrossRef]

37. Oude Elferink, R. P., Meijer, D. K., Kuipers, F., Jansen, P. L., Groen, A. K. and Groothuis, G. M. 1995. Hepatobiliary secretion of organic compounds; molecular mechanisms of membrane transport. Biochim. Biophys. Acta 1241: 215-268. [Medline] [CrossRef]

38. Pan, Y., Omori, K., Ali, I., Tachikawa, M., Terasaki, T., Brouwer, K. L. R. and Nicolazzo, J. A. 2018. Altered expression of small intestinal drug transporters and hepatic metabolic enzymes in a mouse model of familial alzheimer's disease. Mol. Pharm. 15: 4073-4083. [Medline] [CrossRef]

39. Pellock, S. J. and Redinbo, M. R. 2017. Glucuronides in the gut: Sugar-driven symbioses between microbe and host. J. Biol. Chem. 292: 85698576. [Medline] [CrossRef]

40. Pfeiffer, E., Hildebrand, A., Mikula, H. and Metzler, M. 2010. Glucuronidation of zearalenone, zeranol and four metabolites in vitro: formation of glucuronides by various microsomes and human UDP-glucuronosyltransferase isoforms. Mol. Nutr. Food Res. 54: 1468-1476. [Medline] [CrossRef]

41. Pfeiffer, E., Hildebrand, A., Damm, G., Rapp, A., Cramer, B., Humpf, H. U. and Metzler, M. 2009. Aromatic hydroxylation is a major metabolic pathway of the mycotoxin zearalenone in vitro. Mol. Nutr. Food Res. 53: 1123-1133. [Medline] [CrossRef]

42. Prouillac, C., Koraichi, F., Videmann, B., Mazallon, M., Rodriguez, F., Baltas, M. and Lecoeur, S. 2012. In vitro toxicological effects of estrogenic mycotoxins on human placental cells: structure activity relationships. Toxicol. Appl. Pharmacol. 259: 366-375. [Medline] [CrossRef]

43. Reddy, B. S., Engle, A., Simi, B. and Goldman, M. 1992. Effect of dietary fiber on colonic bacterial enzymes and bile acids in relation to colon cancer. Gastroenterology 102: 1475-1482. [Medline] [CrossRef]

44. Righetti, L., Rolli, E., Galaverna, G., Suman, M., Bruni, R. and Dall'Asta, C. 2017. Plant organ cultures as masked mycotoxin biofactories: Deciphering the fate of zearalenone in micropropagated durum wheat roots and leaves. PLoS One 12: e0187247. [Medline] [CrossRef]

45. Rivory, L. P., Riou, J. F., Haaz, M. C., Sable, S., Vuilhorgne, M., Commerçon, A., Pond, S. M. and Robert, J. 1996. Identification and properties of a major plasma metabolite of irinotecan (CPT-11) isolated from the plasma of patients. Cancer Res. 56: 3689-3694. [Medline]

46. Rost, D., Mahner, S., Sugiyama, Y. and Stremmel, W. 2002. Expression and localization of the multidrug resistance-associated protein 3 in rat small and large intestine. Am. J. Physiol. Gastrointest. Liver Physiol. 282: G720-G726. [Medline] [CrossRef]

47. Schneweis, I., Meyer, K., Engelhardt, G. and Bauer, J. 2002. Occurrence of zearalenone-4-beta-D-glucopyranoside in wheat. J. Agric. Food Chem. 50: 1736-1738. [Medline] [CrossRef]

48. Shuster, D. L., Bammler, T. K., Beyer, R. P., Macdonald, J. W., Tsai, J. M., Farin, F. M., Hebert, M. F., Thummel, K. E. and Mao, Q. 2013. Gestational age-dependent changes in gene expression of metabolic enzymes and transporters in pregnant mice. Drug Metab. Dispos. 41: 332-342. [Medline] [CrossRef]

49. Takasuna, K., Hagiwara, T., Hirohashi, M., Kato, M., Nomura, M., Nagai, E., Yokoi, T. and Kamataki, T. 1996. Involvement of beta-glucuronidase in intestinal microflora in the intestinal toxicity of the antitumor camptothecin derivative irinotecan hydrochloride (CPT-11) in rats. Cancer Res. 56: 3752-3757. [Medline]

50. Turgeon, D., Carrier, J. S., Lévesque, E., Hum, D. W. and Bélanger, A. 2001. Relative enzymatic activity, protein stability, and tissue distribution of human steroid-metabolizing UGT2B subfamily members. Endocrinology 142: 778-787. [Medline] [CrossRef]

51. Witaszak, N., Stępień, Ł., Bocianowski, J. and Waśkiewicz, A. 2019. Fusarium species and mycotoxins contaminating veterinary diets for dogs and cats. Microorganisms 7: 7. [Medline] [CrossRef]

52. Yamazaki, M., Suzuki, H. and Sugiyama, Y. 1996. Recent advances in carrier-mediated hepatic uptake and biliary excretion of xenobiotics. Pharm. Res. 13: 497-513. [Medline] [CrossRef]

53. Yang, S., Zhang, H., Sun, F., De Ruyck, K., Zhang, J., Jin, Y., Li, Y., Wang, Z., Zhang, S., De Saeger, S., Zhou, J., Li, Y. and De Boevre, M. 2017. Metabolic profile of zearalenone in liver microsomes from different species and its in vivo metabolism in rats and chickens using ultra high-pressure liquid chromatography-quadrupole/time-of-flight mass spectrometry. J. Agric. Food Chem. 65: 11292-11303. [Medline] [CrossRef]

54. Zhao, F., Li, R., Xiao, S., Diao, H., Viveiros, M. M., Song, X. and Ye, X. 2013. Postweaning exposure to dietary zearalenone, a mycotoxin, promotes premature onset of puberty and disrupts early pregnancy events in female mice. Toxicol. Sci. 132: 431-442. [Medline] [CrossRef]

55. Zielonka, Ł., Waśkiewicz, A., Beszterda, M., Kostecki, M., Dąbrowski, M., Obremski, K., Goliński, P. and Gajęcki, M. 2015. Zearalenone in the intestinal tissues of immature gilts exposed per os to mycotoxins. Toxins (Basel) 7: 3210-3223. [Medline] [CrossRef]

56. Zinedine, A., Soriano, J. M., Moltó, J. C. and Mañes, J. 2007. Review on the toxicity, occurrence, metabolism, detoxification, regulations and intake of zearalenone: an oestrogenic mycotoxin. Food Chem. Toxicol. 45: 1-18. [Medline] [CrossRef] 